\title{
A Finite-Step Injection Problem of Discrete-Time Kalman Filter into the Stationary-State with the Optimal Observation Gain Matrix
}

\author{
Yoshiki TAKEUCHI \\ Dept of Information Science, Osaka Kyoiku University \\ 4-698-1 Asahigaoka, Kashiwara City, Osaka 582-8582, Japan \\ E-mail: takeuti@cc.osaka-kyoiku.ac.jp
}

\begin{abstract}
In this paper, we are concerned with a problem of finding the series of the observation gain matrices by which, in the assigned number of steps, the Kalman filter goes into the stationary state, and which minimizes a quadratic criterion with the same form as the stationary problem. The condition of optimality of the observation gain matrices is obtained in the same form as the corresponding stationary problem. The results of numerical experiments are provided to show the efficiency of the algorithm.
\end{abstract}

\section{Introduction}

The author has already considered the optimization of the observations for the stationary Kalman filter [10]-[19]. In [13], the problem of optimization of the gain matrix of the linear observations for the stationary Kalman filter under the criterion which is quadratic in both the estimation errors and the gain matrix. This result is concerned with the discrete-time systems with time-invariant coefficient matrices. The corresponding problem for continuous-time systems is considered in [18]. For both cases of the discrete-time and continuous-time systems, an efficient condition of optimality is obtained by introducing the change of variables from the observation gain matrix to the set of eigen-vectors and eigen-values of SSNRM (the Symmetric Signal-to-Noise Ratio Matrix), which is a quadratic function of the gain matrix weighted by the inverse of the noise covariance.

By these results, we can obtain the optimal observation gain matrix for the stationary case if we solve the set of equations:

(1) the algebraic matrix Riccati equation for the covariance matrix of the estimation error;

(2) the condition of optimality;

(3) the algebraic matrix Lyapunov equation that accompanies with the condition of optimality; with

(a) the error covariance matrix;

(b) the pair of matrices composed of, respectively, eigen-vectors and eigen-values of SSNRM; (c) a positive definite symmetric matrix which is contained in the condition of optimality; as the variables.

The author also proposed recursive algorithms to solve the above set of equations [19]. Very recently, we noticed that these algorithms are very much similar to that of the fixed-point iteration methods. In fact, by the modification of the algorithms to those of the fixed-point iteration, we got convergences to the optimal values rather rapidly with stable behaviors, for both the discrete-time and continuous-time systems. Thus, we can say that we have already established the method of solution for the optimization of the observations for the stationary Kalman filter.

As you know, when any initial value of the estimation error covariance is given, the stationary Kalman filter behaves non-stationary until it reaches at the stationary state. In fact, the time-evolutions of the estimation error covariance, in this case, are given by the solution of the Riccati difference and differential equations. Especially, in the discrete-time systems, it requires a large number of steps in order to reach the stationary state.

In this paper, for the discrete-time systems with time-invariant coefficients, other than the observation gain, we consider the problem of finding the series of the observation gain matrices by which, in the assigned number of steps, the Kalman filter goes into the stationary state and which minimizes a quadratic criterion with the same form as the stationary case.

Mathematical symbols, in this paper, are used in the following way. $\boldsymbol{R}$ is the space of all real numbers, i.e., $\boldsymbol{R} \triangleq(-\infty, \infty)$. For positive integers $m$ and $n, \boldsymbol{R}^{n}$ and $\boldsymbol{R}^{m \times n}$ denote the spaces of $n$-dimensional vectors and $m \times n$-dimensional matrices whose components take values in $\boldsymbol{R}$. The prime denotes the transpose of a vector or a matrix and the Euclidean norm is $|\cdot|$. Thus, for $x \in \boldsymbol{R}^{n}, \quad|x|=\sqrt{x^{\prime} x}$. The identity matrix of any dimension is denoted by $I$. The components of a matrix are denoted by using subscripts. Thus, $[A]_{i j}$ is the $(i, j)$-component of $A$. In the case where no confusion may arise, we denote $[A]_{i j}$ simply by $a_{i j}$. If $A$ is a 
square matrix, $\operatorname{det}|A|$ and $\operatorname{tr}[A]$ respectively denote the determinant and the trace of $A$. We use $A>0$ and $A \geq 0$ to denote that $A$ is positive definite and nonnegative definite, respectively. The triplet $(\Omega, \mathscr{T}, P)$ is a complete probability space where $\Omega$ is a sample space with elementary events $\omega, \mathscr{F}$ is a $\sigma$-field of subsets of $\Omega$, and $P$ is a probability measure. $E\{\cdot\}$ denotes the expectation and $E\{\cdot \mid \mathscr{G}\}, \mathscr{G} \subset \mathscr{F}$ the conditional expectation, given $\mathscr{G}$, with respect to $P . \sigma\{\cdot\}$ is the minimal sub- $\sigma$-field of $\mathscr{F}$ with respect to which the family of $\mathscr{\mathscr { F }}$-measurable sets or random variables $\{\cdot\}$ is measurable.

\section{Problem Formulation}

Let $\boldsymbol{x} \equiv\left\{x_{t}(\omega) ; t=0,1,2, \cdots\right\}$ denote an $n$-dimensional Gaussian stochastic process given by

$$
\left\{\begin{aligned}
x_{t+1}(\omega) & =A x_{t}(\omega)+G w_{t}(\omega) \\
x_{0}(\omega) & =x^{0}(\omega),
\end{aligned}\right.
$$

where $A \in \boldsymbol{R}^{n \times n}, \quad G \in \boldsymbol{R}^{n \times d_{1}}, \quad x^{0}(\omega)$ is a Gaussian random vector with mean $\hat{x}^{0}$ and covariance $Q^{0}$, and $\boldsymbol{w} \equiv\left\{w_{t}(\omega) ; t=0,1, \cdots\right\} \quad$ is a $d_{1}$ - dimensional standard white Gaussian noise sequence. Suppose that the available data concerned with $\boldsymbol{x}$ is given by the linear observations described by

$$
y_{t}(\omega)=H(t) x_{t}(\omega)+R v_{t}(\omega),
$$

where $\boldsymbol{y} \equiv\left\{y_{t}(\omega) ; t=1,2, \cdots\right\}$ is an $m$-dimensional observation process, $\quad H(t) \in \boldsymbol{R}^{m \times n}, \quad R \in \boldsymbol{R}^{m \times d_{2}}$, and $\boldsymbol{v} \equiv$ $\left\{v_{t}(\omega) ; t=1,2, \cdots\right\}$ is a $d_{2}$-dimensional standard white Gaussian noise sequence. Throughout this paper, we will assume the following two conditions.

(C-1) $G G^{\prime}>0$, and $R_{0} \triangleq R R^{\prime}>0$,

(C-2) $x^{0}(\omega), \boldsymbol{w}$ and $\boldsymbol{v}$ are mutually independent.

It is well-known that the least-squares estimate $\hat{x}_{t}(\omega) \triangleq$ $E\left\{x_{t}(\omega) \mid \mathscr{O}_{t}\right\}$ of $x_{t}(\omega)$ based on $\mathscr{Y}_{t} \triangleq \sigma\left\{y_{s}(\omega) ; s=1,2\right.$, $\cdots, t\}$ is given by the Kalman filter:

$$
\left\{\begin{aligned}
\hat{x}_{t \mid t-1}(\omega) & =A \hat{x}_{t-1 \mid t-1}(\omega), \\
\hat{x}_{t \mid t}(\omega) & =\hat{x}_{t \mid t-1}(\omega)+Q_{t \mid t-1} H^{\prime}(t)\left\{H(t) Q_{t \mid t-1} H^{\prime}(t)+R_{0}\right\}^{-1} \\
& \times\left\{y_{t}(\omega)-H(t) \hat{x}_{t \mid t-1}(\omega)\right\},
\end{aligned}\right.
$$

and

$$
\left\{\begin{aligned}
Q_{t \mid t-1} & =A Q_{t-1 \mid t-1} A^{\prime}+G G^{\prime}, \\
Q_{t \mid t} & =Q_{t \mid t-1}-Q_{t \mid t-1} H^{\prime}(t)\left\{H(t) Q_{t \mid t-1} H^{\prime}(t)+R_{0}\right\}^{-1} H(t) Q_{t \mid t-1},
\end{aligned}\right.
$$

where

$$
Q_{t} \triangleq E\left\{\left[x_{t}(\omega)-\hat{x}_{t}(\omega)\right]\left[x_{t}(\omega)-\hat{x}_{t}(\omega)\right]^{\prime}\right\} .
$$

For the stationary case with $H(t) \equiv H$, we have $Q_{t \mid t-1} \rightarrow Q^{-}$and $Q_{t \mid t} \rightarrow Q$ as $t \rightarrow \infty$, and (3) and (4) are respectively replaced by

$$
\left\{\begin{aligned}
\hat{x}_{t \mid t-1}(\omega) & =A \hat{x}_{t-1 \mid t-1}(\omega), \\
\hat{x}_{t \mid t}(\omega)= & \hat{x}_{t \mid t-1}(\omega)+Q^{-} H^{\prime}\left\{H Q^{-} H^{\prime}+R_{0}\right\}^{-1} \\
& \times\left\{y_{t}(\omega)-H \hat{x}_{t \mid t-1}(\omega)\right\},
\end{aligned}\right.
$$

and

$$
\left\{\begin{array}{l}
Q^{-}=A Q A^{\prime}+G G^{\prime} \\
Q=Q^{-}-Q^{-} H^{\prime}\left\{H Q^{-} H^{\prime}+R_{0}\right\}^{-1} H Q^{-} .
\end{array}\right.
$$

For this case, we already obtained the following result for the optimal value of the observation gain matrix $H$.

[Problem 1] Let $M \in \boldsymbol{R}^{n \times n}$ and $N \in \boldsymbol{R}^{n \times n} \quad$ be symmetric positive-definite matrices. Find $H \in \boldsymbol{R}^{m \times n}$ such that

$$
J=\operatorname{tr}[M Q]+\operatorname{tr}\left[H N H^{\prime}\right] \rightarrow \min .
$$

«Theorem $1 »^{[13]}$ Let $\tilde{m} \triangleq \operatorname{rank}[H]$. Let us denote as

$$
N^{1 / 2} H^{\prime} R_{0}^{-1} H N^{1 / 2}=\tilde{U} \tilde{\Xi} \tilde{U}^{\prime},
$$

where $\tilde{U} \in \boldsymbol{R}^{n \times \tilde{m}}$ denotes a set of $\tilde{m}$ - column vectors of an orthogonal matrix $U \in \boldsymbol{R}^{n \times n}$ such that $\tilde{U}^{\prime} \tilde{U}=I$ and $\tilde{\Xi}=\operatorname{diag}\left(\xi_{1}, \xi_{2}, \cdots, \xi_{\tilde{m}}\right), \xi_{1} \geq \xi_{2} \geq \cdots \geq \xi_{\tilde{m}}>0$. Also, let

$$
\begin{gathered}
R_{0}=\left[\begin{array}{cc}
\tilde{\Gamma} & \bar{\Gamma}
\end{array}\right] \Psi\left[\begin{array}{l}
\tilde{\Gamma}^{\prime} \\
\bar{\Gamma}^{\prime}
\end{array}\right], \quad \Psi=\operatorname{diag}\left(\psi_{1}, \psi_{2}, \cdots, \psi_{\tilde{m}}, \cdots, \psi_{m}\right), \\
\psi_{1} \leq \psi_{2} \leq \cdots \leq \psi_{\tilde{m}} \leq \cdots \leq \psi_{m} .
\end{gathered}
$$

In addition to (C-1) and (C-2), assume that

(C-3) The set $\mathscr{H}$ defined by

$$
\begin{aligned}
& \mathscr{H} \triangleq\{(\tilde{U}, \tilde{\Xi}) ;(7) \text { has a solution } Q>0 \\
& \left.\quad \text { with a stable matrix } F \triangleq Q\left(Q^{-}\right)^{-1} A\right\} \\
& \text { is not empty. }
\end{aligned}
$$

Then, the condition of optimality for Problem 1 is given

$$
N^{-1 / 2} Q X Q N^{-1 / 2} \tilde{U}=\tilde{U} \tilde{\Psi},
$$

where $\tilde{\Gamma} \in \boldsymbol{R}^{m \times \tilde{m}}, \quad \tilde{\Psi} \triangleq \operatorname{diag}\left(\psi_{1}, \psi_{2}, \cdots, \psi_{\tilde{m}}\right)$ and $X \in \boldsymbol{R}^{n \times n}$ is a solution of

$$
X=F^{\prime} X F+M .
$$

The optimal value of the stationary observation gain matrix is given by

$$
H=\tilde{\Gamma} \tilde{\Psi}^{1 / 2} \tilde{\Xi}^{1 / 2} \tilde{U}^{\prime} N^{-1 / 2}
$$
lem:

In this paper, we are concerned with the following prob-

[Problem 2] For any given initial value $Q_{0 \mid 0}=Q^{0}>Q$ and the injection time defined by $\tau \triangleq \min _{t}\left\{Q_{t \mid t}=Q\right\}$, find, 
under the Riccati equation (4), the optimal sequence $H(t) \in \boldsymbol{R}^{m \times n}, t=1,2, \cdots, \tau$ of the observation gain matrices which minimizes

$$
J_{\tau} \triangleq \sum_{t=1}^{\tau}\left\{\operatorname{tr}\left[M Q_{t \mid t}\right]+\operatorname{tr}\left[H(t) N H^{\prime}(t)\right]\right\} .
$$

\section{Main Results}

For Problem 2, we have the following result.

«Theorem 2» Assume (C-1)-(C-3). Let us denote as

$$
N^{1 / 2} H^{\prime}(t) R_{0}^{-1} H(t) N^{1 / 2}=\tilde{U}(t) \tilde{\Xi}(t) \tilde{U}^{\prime}(t),
$$

$$
\begin{gathered}
\tilde{U}^{\prime}(t) \tilde{U}(t)=I, \quad \tilde{\Xi}(t)=\operatorname{diag}\left\{\xi_{1}(t), \xi_{2}(t), \cdots, \xi_{\tilde{m}}(t)\right\}, \\
\xi_{1}(t) \geq \xi_{2}(t) \geq \cdots \geq \xi_{\tilde{m}}(t)>0 .
\end{gathered}
$$

Then, the optimal sequence $H(t) \in \boldsymbol{R}^{m \times n}, t=1,2, \cdots, \tau$ is given by

$$
H(t)=\tilde{\Gamma} \tilde{\Psi}^{1 / 2} \tilde{\Xi}^{1 / 2}(t) \tilde{U}^{\prime}(t) N^{-1 / 2},
$$

and the condition of optimality for Problem 2 is given by

$$
\begin{gathered}
N^{-1 / 2} Q_{t \mid t} X(t) Q_{t \mid t} N^{-1 / 2} \tilde{U}(t)=\tilde{U}(t) \tilde{\Psi}, \\
t=1,2, \cdots, \tau-1,
\end{gathered}
$$

where $X(t) \in \boldsymbol{R}^{n \times n}, t=1,2, \cdots, \tau-1$, is given by

$$
\left\{\begin{array}{l}
X(t)=F^{\prime}(t+1) X(t+1) F(t+1)+M, \\
X(\tau) \triangleq Q^{-1} N^{1 / 2} \tilde{U}(\tau) \tilde{\Psi} \tilde{U}^{\prime}(\tau) N^{1 / 2} Q^{-1},
\end{array}\right.
$$

and

$$
F(t) \triangleq Q_{t \mid t} Q_{t \mid t-1}^{-1} A .
$$

[Remark 1] For $t=0$, the values of $Q^{0}=Q_{0 \mid 0}$ and $X(0)$ need not satisfy the condition (16). However, for $t=\tau$, we have the relation (16), since the second relation of (17) implies

$$
N^{-1 / 2} Q X(\tau) Q N^{-1 / 2}=\tilde{U}(\tau) \tilde{\Psi} \tilde{U}^{\prime}(\tau) .
$$

[Remark 2] By the first relation of (17), we have $X(t)>0$ for $t=1,2, \cdots, \tau-1$. However, the definition of $X(\tau)$ in (17) implies, $X(\tau)$ is a non-negative definite but singular matrix for the case $\tilde{m}<n$.

For the proof of Theorem 2, we need the following lemmas.

«Lemma 1» Assume (C-1)-(C-3). Then, for any $t \in\{1,2, \cdots, \tau\}$, we have the following (i)-(iii).

(i) For $t \leq \tau-1$,

$$
\begin{aligned}
\frac{\partial \tilde{\Xi}(\tau)}{\partial u_{i j}(t)}= & \tilde{U}^{\prime}(\tau) N^{1 / 2}\left(Q_{\tau \mid \tau-1}\right)^{-1} A \frac{\partial Q_{\tau-1 \mid \tau-1}}{\partial u_{i j}(t)} A^{\prime}\left(Q_{\tau \mid \tau-1}\right)^{-1} N^{1 / 2} \tilde{U}(\tau) \\
& -\tilde{U}^{\prime}(\tau) \frac{\partial \tilde{U}(\tau)}{\partial u_{i j}(t)} \tilde{\Xi}(\tau)-\tilde{\Xi}(\tau) \frac{\partial \tilde{U}^{\prime}(\tau)}{\partial u_{i j}(t)} \tilde{U}(\tau)
\end{aligned}
$$

$$
\begin{aligned}
\frac{\partial \tilde{\Xi}(\tau)}{\partial \xi_{i}(t)}= & \tilde{U}^{\prime}(\tau) N^{1 / 2}\left(Q_{\tau \mid \tau-1}\right)^{-1} A \frac{\partial Q_{\tau-1 \mid \tau-1}}{\partial \xi_{i}(t)} A^{\prime}\left(Q_{\tau \mid \tau-1}\right)^{-1} N^{1 / 2} \tilde{U}(\tau) \\
& -\tilde{U}^{\prime}(\tau) \frac{\partial \tilde{U}(\tau)}{\partial \xi_{i}(t)} \tilde{\Xi}(\tau)-\tilde{\Xi}(\tau) \frac{\partial \tilde{U}^{\prime}(\tau)}{\partial \xi_{i}(t)} \tilde{U}(\tau)
\end{aligned}
$$

(ii) For $t+1 \leq \sigma \leq \tau-1$,

$$
\begin{aligned}
& \frac{\partial Q_{\sigma \mid \sigma}}{\partial u_{i j}(t)}=F(\sigma) \frac{\partial Q_{\sigma-1 \mid \sigma-1}}{\partial u_{i j}(t)} F^{\prime}(\sigma) \\
& -Q_{\sigma \mid \sigma} N^{-1 / 2}\left[\frac{\partial}{\partial u_{i j}(t)}\left\{\tilde{U}(\sigma) \tilde{\Xi}(\sigma) \tilde{U}^{\prime}(\sigma)\right\}\right] N^{-1 / 2} Q_{\sigma \mid \sigma} \\
& \frac{\partial Q_{\sigma \mid \sigma}}{\partial \xi_{i}(t)}=F(\sigma) \frac{\partial Q_{\sigma-1 \mid \sigma-1}}{\partial \xi_{i}(t)} F^{\prime}(\sigma) \\
& -Q_{\sigma \mid \sigma} N^{-1 / 2}\left[\frac{\partial}{\partial \xi_{i}(t)}\left\{\tilde{U}(\sigma) \tilde{\Xi}(\sigma) \tilde{U}^{\prime}(\sigma)\right\}\right] N^{-1 / 2} Q_{\sigma \mid \sigma}
\end{aligned}
$$

(iii) For $\sigma=t$,

$$
\begin{aligned}
& \frac{\partial Q_{t \mid t}}{\partial u_{i j}(t)}=-Q_{t \mid t} N^{-1 / 2}\left\{E_{i j} \tilde{\Xi}(t) \tilde{U}^{\prime}(t)+\tilde{U}(t) \tilde{\Xi}(t) E_{j i}\right\} N^{-1 / 2} Q_{t \mid t}, \\
& \frac{\partial Q_{t \mid t}}{\partial \xi_{i}(t)}=-Q_{t \mid t} N^{-1 / 2} \tilde{U}(t) E_{i i} \tilde{U}^{\prime}(t) N^{-1 / 2} Q_{t \mid t}
\end{aligned}
$$

where

$$
E_{i j} \triangleq\left[\begin{array}{ccc} 
& j & \\
0 & 0 & 0 \\
& \vdots & 0 \\
0 \cdots \cdots & 1 & 0 \\
& 0 & \\
0 & \vdots & 0 \\
0 & 0 &
\end{array}\right] i
$$

(Proof) First, let us show (20) and (21). By applying the well-known formula of differentials of an inverse matrix:

$$
\frac{\partial Z^{-1}}{\partial u_{i j}}=-Z^{-1} \frac{\partial Z}{\partial u_{i j}} Z^{-1},
$$

with $Z=Q_{\tau \mid \tau}^{-1}$, we have

$$
\frac{\partial Q_{\tau \mid \tau}}{\partial u_{i j}(t)}=\frac{\partial\left(Q_{\tau \mid \tau}^{-1}\right)^{-1}}{\partial u_{i j}(t)}=-Q_{\tau \mid \tau} \frac{\partial Q_{\tau \mid \tau}^{-1}}{\partial u_{i j}(t)} Q_{\tau \mid \tau},
$$

Note that we have

$$
Q_{\tau \mid \tau}^{-1}=Q_{\tau \mid \tau-1}^{-1}+N^{-1 / 2} \tilde{U}(\tau-1) \tilde{\Xi}(\tau-1) \tilde{U}^{\prime}(\tau-1) N^{-1 / 2},
$$

since $H^{\prime}(t) R_{0}^{-1} H(t)=N^{-1 / 2} \tilde{U}(t) \tilde{\Xi}(t) \tilde{U}^{\prime}(t) N^{-1 / 2}$. Then, substitution of (29) into the right-hand side of (28) yields 


$$
\begin{aligned}
\frac{\partial Q_{\tau \mid \tau}}{\partial u_{i j}(t)} & =-Q_{\tau \mid \tau} \frac{\partial Q_{|\tau| \tau-1}^{-1}}{\partial u_{i j}(t)} Q_{\tau \mid \tau} \\
& -Q_{\tau \mid \tau} N^{-1 / 2}\left[\frac{\partial}{\partial u_{i j}(t)}\left\{\tilde{U}(\tau) \tilde{\Xi}(\tau) \tilde{U}^{\prime}(\tau)\right\}\right] N^{-1 / 2} Q_{\tau \mid \tau} .
\end{aligned}
$$

Since the first relation of (4) implies

$$
\frac{\partial Q_{\tau \mid \tau-1}}{\partial u_{i j}(t)}=A \frac{\partial Q_{\tau-1 \mid \tau-1}}{\partial u_{i j}(t)} A^{\prime},
$$

and since $Q_{\tau \mid \tau}=Q$, we have

$$
\begin{aligned}
& \frac{\partial Q_{\tau \mid \tau}}{\partial u_{i j}(t)}=0=F(\tau) \frac{\partial Q_{\tau-1 \mid \tau-1}}{\partial u_{i j}(t)} F^{\prime}(\tau) \\
& -Q N^{-1 / 2}\left[\frac{\partial}{\partial u_{i j}(t)}\left\{\tilde{U}(\tau) \tilde{\Xi}(\tau) \tilde{U}^{\prime}(\tau)\right\}\right] N^{-1 / 2} Q .
\end{aligned}
$$

Since $F(\tau) \triangleq Q_{\tau \mid \tau}\left(Q_{\tau \mid \tau-1}\right)^{-1} A=Q\left(Q_{\tau \mid \tau-1}\right)^{-1} A$ and $Q_{\tau \mid \tau}=Q$ $>0$, it follows from (32) that

$$
\begin{aligned}
& \left(Q_{\tau \mid \tau-1}\right)^{-1} A \frac{\partial Q_{\tau-1 \mid \tau-1}}{\partial u_{i j}(t)} A^{\prime}\left(Q_{\tau \mid \tau-1}\right)^{-1} \\
& =N^{-1 / 2}\left[\frac{\partial}{\partial u_{i j}(t)}\left\{\tilde{U}(\tau) \tilde{\Xi}(\tau) \tilde{U}^{\prime}(\tau)\right\}\right] N^{-1 / 2} .
\end{aligned}
$$

Multiplying $\tilde{U}^{\prime}(\tau) N^{1 / 2}$ from left and $N^{1 / 2} \tilde{U}(\tau)$ from right to both sides of (33), we have (20). For (21), we have, by the same way as we obtained (32),

$$
\begin{aligned}
& \frac{\partial Q_{\tau \mid \tau}}{\partial \xi_{i}(t)}=0=F(\tau) \frac{\partial Q_{\tau-1 \mid \tau-1}}{\partial \xi_{i}(t)} F^{\prime}(\tau) \\
& -Q N^{-1 / 2}\left[\frac{\partial}{\partial \xi_{i}(t)}\left\{\tilde{U}(\tau) \tilde{\Xi}(\tau) \tilde{U}^{\prime}(\tau)\right\}\right] N^{-1 / 2} Q,
\end{aligned}
$$

which implies (21).

Next, let us show (22) and (23). By the same way as we obtained (32), we have (22). Also, the same way used in obtaining (34) yields (23).

Finally, when $\sigma=t$, it is clear that (22) and (23) respectively reduce (24) and (25). This completes the proof.

«Lemma 2» Assume (C-1)-(C-3). Also, assume that the following three relations hold for any $t \in\{1,2, \cdots, \tau\}$ and $t+1 \leq \sigma \leq \tau-1$.

$$
\begin{gathered}
X(\sigma)=F^{\prime}(\sigma+1) X(\sigma+1) F(\sigma+1)+M, \\
F(\sigma) \triangleq Q_{\sigma \mid \sigma} Q_{\mid \sigma \sigma-1}^{-1} A, \\
N^{-1 / 2} Q_{\sigma \mid \sigma} X(\sigma) Q_{\sigma \mid \sigma} N^{-1 / 2} \tilde{U}(\sigma)=\tilde{U}(\sigma) \tilde{\Psi},
\end{gathered}
$$

Then, for $t+1 \leq \sigma \leq \tau-1$, we have

$$
\begin{aligned}
& \operatorname{tr}\left[X(\sigma) \frac{\partial Q_{\sigma \mid \sigma}}{\partial u_{i j}(t)}\right]= \operatorname{tr}\left[F^{\prime}(\sigma) X(\sigma) F(\sigma) \frac{\partial Q_{\sigma-1 \mid \sigma-1}}{\partial u_{i j}(t)}\right] \\
&-\operatorname{tr}\left[\tilde{\Psi} \frac{\partial \tilde{\Xi}(\sigma)}{\partial u_{i j}(t)}\right],
\end{aligned}
$$

$$
\begin{aligned}
\operatorname{tr}\left[X(\sigma) \frac{\partial Q_{\sigma \mid \sigma}}{\partial \xi_{i}(t)}\right] & =\operatorname{tr}\left[F^{\prime}(\sigma) X(\sigma) F(\sigma) \frac{\partial Q_{\sigma-1 \mid \sigma-1}}{\partial \xi_{i}(t)}\right] \\
& -\operatorname{tr}\left[\tilde{\Psi} \frac{\partial \tilde{\Xi}(\sigma)}{\partial \xi_{i}(t)}\right] .
\end{aligned}
$$

(Proof) Substituting (22) into the left-hand side of (38), we have

$$
\begin{array}{r}
\operatorname{tr}\left[X(\sigma) \frac{\partial Q_{\sigma \mid \sigma}}{\partial u_{i j}(t)}\right]=\operatorname{tr}\left[X(\sigma) F(\sigma) \frac{\partial Q_{\sigma-|| \sigma-1}}{\partial u_{i j}(t)} F^{\prime}(\sigma)\right] \\
-\operatorname{tr}\left[\tilde{U}^{\prime}(\sigma) N^{-1 / 2} Q_{\sigma \mid \sigma} X(\sigma) Q_{\sigma \mid \sigma} N^{-1 / 2} \frac{\partial \tilde{U}(\sigma)}{\partial u_{i j}(t)} \tilde{\Xi}(\sigma)\right] \\
-\operatorname{tr}\left[\tilde{\Xi}(\sigma) \frac{\partial \tilde{U}^{\prime}(\sigma)}{\partial u_{i j}(t)} N^{-1 / 2} Q_{\sigma \mid \sigma} X(\sigma) Q_{\sigma \mid \sigma} N^{-1 / 2} \tilde{U}(\sigma)\right] \\
-\operatorname{tr}\left[\tilde{U}^{\prime}(\sigma) N^{-1 / 2} Q_{\sigma \mid \sigma} X(\sigma) Q_{\sigma \mid \sigma} N^{-1 / 2} \tilde{U}(\sigma) \frac{\partial \tilde{\Xi}(\sigma)}{\partial u_{i j}(t)}\right],
\end{array}
$$

where we have used the relation $\operatorname{tr}[A B]=\operatorname{tr}[B A]$ in the last three terms in the right-hand side. Note that because of (37), we have for the sum of the second and third terms in (40),

$$
\begin{aligned}
& -\operatorname{tr}\left[\tilde{U}^{\prime}(\sigma) N^{-1 / 2} Q_{\sigma \mid \sigma} X(\sigma) Q_{\sigma \mid \sigma} N^{-1 / 2} \frac{\partial \tilde{U}(\sigma)}{\partial u_{i j}(t)} \tilde{\Xi}(\sigma)\right] \\
& -\operatorname{tr}\left[\tilde{\Xi}(\sigma) \frac{\partial \tilde{U}^{\prime}(\sigma)}{\partial u_{i j}(t)} N^{-1 / 2} Q_{\sigma \mid \sigma} X(\sigma) Q_{\sigma \mid \sigma} N^{-1 / 2} \tilde{U}(\sigma)\right] \\
& =-\operatorname{tr}\left[\tilde{\Psi} \tilde{U}^{\prime}(\sigma) \frac{\partial \tilde{U}(\sigma)}{\partial u_{i j}(t)} \tilde{\Xi}(\sigma)\right]-\operatorname{tr}\left[\tilde{\Xi}(\sigma) \frac{\partial \tilde{U}^{\prime}(\sigma)}{\partial u_{i j}(t)} \tilde{U}(\sigma) \tilde{\Psi}\right] \\
& =-\operatorname{tr}\left[\tilde{U}^{\prime}(\sigma) \frac{\partial \tilde{U}(\sigma)}{\partial u_{i j}(t)} \tilde{\Xi}(\sigma) \tilde{\Psi}\right]-\operatorname{tr}\left[\frac{\partial \tilde{U}^{\prime}(\sigma)}{\partial u_{i j}(t)} \tilde{U}(\sigma) \tilde{\Psi} \tilde{\Xi}(\sigma)\right] \\
& =-\operatorname{tr}\left[\left\{\tilde{U}^{\prime}(\sigma) \frac{\partial \tilde{U}(\sigma)}{\partial u_{i j}(t)}+\frac{\partial \tilde{U}^{\prime}(\sigma)}{\partial u_{i j}(t)} \tilde{U}(\sigma)\right\} \tilde{\Psi} \tilde{\Xi}(\sigma)\right] \\
& =0,
\end{aligned}
$$

where we have used the relation $\tilde{\Psi} \tilde{\Xi}(\sigma)=\tilde{\Xi}(\sigma) \tilde{\Psi}$ in the third equality. Also by (37), it is easily seen for the fourth term in (40) that

$$
\begin{aligned}
& -\operatorname{tr}\left[\tilde{U}^{\prime}(\sigma) N^{-1 / 2} Q_{\sigma \mid \sigma} X(\sigma) Q_{\sigma \mid \sigma} N^{-1 / 2} \tilde{U}(\sigma) \frac{\partial \tilde{\Xi}(\sigma)}{\partial u_{i j}(t)}\right] \\
& =-\operatorname{tr}\left[\tilde{\Psi} \frac{\partial \tilde{\Xi}(\sigma)}{\partial u_{i j}(t)}\right] .
\end{aligned}
$$

Substituting (41) and (42) into (40), we have (38). The relation (39) is obtained in the same way except that $u_{i j}(t)$ is replaced by $\xi_{i}(t)$. This completes the proof.

In advance of the proof of Theorem 2, we now show that Problem 2 is described in the dynamic programing frame- 
work as follows.

[Problem 3 (Dynamic Programing Formulation)] Let us denote the performance criterion for the partial problem with the time interval $\{t, t+1, \cdots, \tau\}$ by

$$
J_{t}^{\tau} \triangleq \sum_{\sigma=t}^{\tau}\left\{\operatorname{tr}\left[M Q_{\sigma \mid \sigma}\right]+\operatorname{tr}[\tilde{\Psi} \tilde{\Xi}(\sigma)]\right\},
$$

and the optimal value of $J_{t}^{\tau}$ by

$$
\begin{aligned}
V_{t}^{\tau} & \equiv V_{t}^{\tau}(\tilde{U}, \tilde{\Xi}) \\
& \triangleq \min _{\substack{U(\sigma), \tilde{\Xi}(\sigma) \\
t \leq \sigma \leq \tau-1}}\left\{J_{t}^{\tau} \mid \tilde{U}^{\prime}(\sigma) \tilde{U}(\sigma)=I, t \leq \sigma \leq \tau\right\} .
\end{aligned}
$$

Then, under the relations:

$$
Q_{\sigma \mid \sigma-1}=A Q_{\sigma-1 \mid \sigma-1} A^{\prime}+G_{0},
$$

and

$$
Q_{\sigma \mid \sigma}{ }^{-1}=Q_{\sigma \mid \sigma-1}{ }^{-1}+N^{-1 / 2} \tilde{U}(\sigma) \tilde{\Xi}(\sigma) \tilde{U}^{\prime}(\sigma) N^{-1 / 2},
$$

find $V_{t}^{\tau}$ and $\left(\tilde{U}^{\prime}(\sigma), \tilde{\Xi}(\sigma)\right), t \leq \sigma \leq \tau$ for all $t=\tau-1$, $\tau-2, \cdots, 1$.

For Problem 3, we have the following lemmas.

«Lemma 3» Assume (C-1)-(C-3). Let us denote

$$
J_{t} \triangleq \operatorname{tr}\left[M Q_{t \mid t}\right]+\operatorname{tr}[\tilde{\Psi} \tilde{\Xi}(t)] .
$$

The Bellman's equation for Problem 3 is described by

$$
V_{t}^{\tau}(\tilde{U}, \tilde{\Xi})=\min _{\tilde{U}(t), \tilde{\Xi}(t)}\left\{V_{t+1}^{\tau}(\tilde{U}, \tilde{\Xi})+J_{t} \mid \tilde{U}^{\prime}(t) \tilde{U}(t)=I\right\} .
$$

«Lemma 4» Assume (C-1)-(C-3). Also, assume that (35)-(37) hold for any $t \in\{1,2, \cdots, \tau\}$ and $t+1 \leq \sigma \leq \tau-1$. Then, for $t+1 \leq \sigma \leq \tau-1$, we have

$$
\begin{gathered}
\frac{\partial V_{\sigma}^{\tau}}{\partial u_{i j}(t)}=\operatorname{tr}\left[F^{\prime}(\sigma) X(\sigma) F(\sigma) \frac{\partial Q_{\sigma-1 \mid \sigma-1}}{\partial u_{i j}(t)}\right], \\
\frac{\partial V_{\sigma}^{\tau}}{\partial \xi_{i}(t)}=\operatorname{tr}\left[F^{\prime}(\sigma) X(\sigma) F(\sigma) \frac{\partial Q_{\sigma-1 \mid \sigma-1}}{\partial \xi_{i}(t)}\right] .
\end{gathered}
$$

In addition, we have

$$
\begin{gathered}
\frac{\partial}{\partial u_{i j}(t)}\left\{V_{t+1}^{\tau}+J_{t}\right\}=\operatorname{tr}\left[X(t) \frac{\partial Q_{t \mid t}}{\partial u_{i j}(t)}\right], \\
\frac{\partial}{\partial \xi_{i}(t)}\left\{V_{t+1}^{\tau}+J_{t}\right\}=\operatorname{tr}\left[X(t) \frac{\partial Q_{t \mid t}}{\partial \xi_{i}(t)}\right]+\psi_{i} .
\end{gathered}
$$

(Proof) First, let us show (50). Since $Q_{\tau \mid \tau}=Q$, (43) and (44) with $t<\sigma=\tau-1$ implies

$$
\begin{aligned}
& \frac{\partial V_{\tau-1}^{\tau}}{\partial u_{i j}(t)}=\frac{\partial}{\partial u_{i j}(t)} \sum_{\sigma=\tau-1}^{\tau}\left\{\operatorname{tr}\left[M Q_{\sigma \mid \sigma}\right]+\operatorname{tr}[\tilde{\Psi} \tilde{\Xi}(\sigma)]\right\} \\
& =\operatorname{tr}\left[M \frac{\partial Q_{\tau-1 \mid \tau-1}}{\partial u_{i j}(t)}\right]+\operatorname{tr}\left[\tilde{\Psi} \frac{\partial \tilde{\Xi}(\tau-1)}{\partial u_{i j}(t)}\right]+\operatorname{tr}\left[\tilde{\Psi} \frac{\partial \tilde{\Xi}(\tau)}{\partial u_{i j}(t)}\right] .
\end{aligned}
$$

Noting (20) and (17), we see that

$$
\begin{aligned}
& \operatorname{tr}\left[\tilde{\Psi} \frac{\partial \tilde{\Xi}(\tau)}{\partial u_{i j}(t)}\right] \\
& =\operatorname{tr}\left[\tilde{\Psi} \tilde{U}^{\prime}(\tau) N^{1 / 2}\left(Q_{\tau \mid \tau-1}\right)^{-1} A \frac{\partial Q_{\tau-1 \mid \tau-1}}{\partial u_{i j}(t)} A^{\prime}\left(Q_{\tau \mid \tau-1}\right)^{-1} N^{1 / 2} \tilde{U}(\tau)\right] \\
& -\operatorname{tr}\left[\tilde{\Psi}\left\{\tilde{U}^{\prime}(\tau) \frac{\partial \tilde{U}(\tau)}{\partial u_{i j}(t)} \tilde{\Xi}(\tau)+\tilde{\Xi}(\tau) \frac{\partial \tilde{U}^{\prime}(\tau)}{\partial u_{i j}(t)} \tilde{U}(\tau)\right\}\right] \\
& =\operatorname{tr}\left[A^{\prime}\left(Q_{\tau \mid \tau-1}\right)^{-1} N^{1 / 2} \tilde{U}(\tau) \tilde{\Psi} \tilde{U}^{\prime}(\tau) N^{1 / 2}\left(Q_{\tau \mid \tau-1}\right)^{-1} A \frac{\partial Q_{\tau-1 \mid \tau-1}}{\partial u_{i j}(t)}\right] \\
& -\operatorname{tr}\left[\left\{\tilde{U}^{\prime}(\tau) \frac{\partial \tilde{U}(\tau)}{\partial u_{i j}(t)}+\frac{\partial \tilde{U}^{\prime}(\tau)}{\partial u_{i j}(t)} \tilde{U}(\tau)\right\} \tilde{\Xi}(\tau) \tilde{\Psi}\right] \\
& =\operatorname{tr}\left[F^{\prime}(\tau) X(\tau) F(\tau) \frac{\partial Q_{\tau-1 \mid \tau-1}}{\partial u_{i j}(t)}\right],
\end{aligned}
$$

where the first equality follows from (20), the second one from $\operatorname{tr}[A B]=\operatorname{tr}[B A]$ and the last one from (17) and $\tilde{U}^{\prime}(\tau) \tilde{U}(\tau)=I$. Thus, the sum of the first and third terms in the last expression of (54) is computed as

$$
\begin{aligned}
& \operatorname{tr}\left[M \frac{\partial Q_{\tau-1 \mid \tau-1}}{\partial u_{i j}(t)}\right]+\operatorname{tr}\left[\tilde{\Psi} \frac{\partial \tilde{\Xi}(\tau)}{\partial u_{i j}(t)}\right] \\
& =\operatorname{tr}\left[M \frac{\partial Q_{\tau-1 \mid \tau-1}}{\partial u_{i j}(t)}\right]+\operatorname{tr}\left[F^{\prime}(\tau) X(\tau) F(\tau) \frac{\partial Q_{\tau-1 \mid \tau-1}}{\partial u_{i j}(t)}\right] \\
& =\operatorname{tr}\left[\left\{F^{\prime}(\tau) X(\tau) F(\tau)+M\right\} \frac{\partial Q_{\tau-1 \mid \tau-1}}{\partial u_{i j}(t)}\right] \\
& =\operatorname{tr}\left[X(\tau-1) \frac{\partial Q_{\tau-1 \mid \tau-1}}{\partial u_{i j}(t)}\right] .
\end{aligned}
$$

Using (38) with $\sigma=\tau-1$ to the last expression in (56), we have

$$
\begin{aligned}
& \operatorname{tr}\left[M \frac{\partial Q_{\tau-1 \mid \tau-1}}{\partial u_{i j}(t)}\right]+\operatorname{tr}\left[\tilde{\Psi} \frac{\partial \tilde{\Xi}(\tau)}{\partial u_{i j}(t)}\right] \\
& =\operatorname{tr}\left[F^{\prime}(\tau-1) X(\tau-1) F(\tau-1) \frac{\partial Q_{\tau-2 \mid \tau-2}}{\partial u_{i j}(t)}\right] \\
& -\operatorname{tr}\left[\tilde{\Psi} \frac{\partial \tilde{\Xi}(\tau-1)}{\partial u_{i j}(t)}\right] .
\end{aligned}
$$

Substituting (57) into (54), we have

$$
\frac{\partial V_{\tau-1}^{\tau}}{\partial u_{i j}(t)}=\operatorname{tr}\left[F^{\prime}(\tau-1) X(\tau-1) F(\tau-1) \frac{\partial Q_{\tau-2 \mid \tau-2}}{\partial u_{i j}(t)}\right],
$$

which is (50) for $\sigma=\tau-1$. Next, if we have (50) for $\sigma=s+1$, i.e. 


$$
\frac{\partial V_{s+1}^{\tau}}{\partial u_{i j}(t)}=\operatorname{tr}\left[F^{\prime}(s+1) X(s+1) F(s+1) \frac{\partial Q_{s \mid s}}{\partial u_{i j}(t)}\right],
$$

It follows that

$$
\begin{aligned}
\frac{\partial V_{s}^{\tau}}{\partial u_{i j}(t)} & =\frac{\partial V_{s+1}^{\tau}}{\partial u_{i j}(t)}+\operatorname{tr}\left[M \frac{\partial Q_{s \mid s}}{\partial u_{i j}(t)}\right]+\operatorname{tr}\left[\tilde{\Psi} \frac{\partial \tilde{\Xi}(s)}{\partial u_{i j}(t)}\right] \\
& =\operatorname{tr}\left[F^{\prime}(s+1) X(s+1) F(s+1) \frac{\partial Q_{s \mid s}}{\partial u_{i j}(t)}\right] \\
& +\operatorname{tr}\left[M \frac{\partial Q_{s \mid s}}{\partial u_{i j}(t)}\right]+\operatorname{tr}\left[\tilde{\Psi} \frac{\partial \tilde{\Xi}(s)}{\partial u_{i j}(t)}\right] \\
& =\operatorname{tr}\left[\left\{F^{\prime}(s+1) X(s+1) F(s+1)+M\right\} \frac{\partial Q_{s \mid s}}{\partial u_{i j}(t)}\right] \\
& +\operatorname{tr}\left[\tilde{\Psi} \frac{\partial \tilde{\Xi}(s)}{\partial u_{i j}(t)}\right] \\
& =\operatorname{tr}\left[X(s) \frac{\partial Q_{s \mid s}}{\partial u_{i j}(t)}\right]+\operatorname{tr}\left[\tilde{\Psi} \frac{\partial \tilde{\Xi}(s)}{\partial u_{i j}(t)}\right] \\
& =\operatorname{tr}\left[F^{\prime}(s) X(s) F(s) \frac{\partial Q_{s-1 \mid s-1}}{\partial u_{i j}(t)}\right],
\end{aligned}
$$

where we have used (38) with $\sigma=s$ in the last equality. Thus, we have (50) for $t+1 \leq \sigma \leq \tau-1$.

Next, let us show (52). From (50) for $\sigma=t+1$, we have

$$
\frac{\partial V_{t+1}^{\tau}}{\partial u_{i j}(t)}=\operatorname{tr}\left[F^{\prime}(t+1) X(t+1) F(t+1) \frac{\partial Q_{t \mid t}}{\partial u_{i j}(t)}\right] .
$$

Hence, we see

$$
\begin{aligned}
& \frac{\partial}{\partial u_{i j}(t)}\left\{V_{t+1}^{\tau}+J_{t}\right\} \\
& =\operatorname{tr}\left[F^{\prime}(t+1) X(t+1) F(t+1) \frac{\partial Q_{t \mid t}}{\partial u_{i j}(t)}\right] \\
& +\operatorname{tr}\left[M \frac{\partial Q_{t \mid t}}{\partial u_{i j}(t)}\right]+\operatorname{tr}\left[\tilde{\Psi} \frac{\partial \tilde{\Xi}(t)}{\partial u_{i j}(t)}\right] \\
& =\operatorname{tr}\left[\left\{F^{\prime}(t+1) X(t+1) F(t+1)+M\right\} \frac{\partial Q_{t \mid t}}{\partial u_{i j}(t)}\right] \\
& =\operatorname{tr}\left[X(t) \frac{\partial Q_{t \mid t}}{\partial u_{i j}(t)}\right],
\end{aligned}
$$

where the fact $\partial \tilde{\Xi}(t) / \partial u_{i j}(t)=0$ is used in the second equality. Thus, we have (52). Similarly, (51) and (53) follow if we use (39) instead of (38).

(Proof of Theorem 2) First, let us define the Lagrangean

$$
\begin{aligned}
& L(\tilde{U}(t), \tilde{\Xi}(t), \tilde{\Lambda}(t)) \\
& \triangleq V_{t+1}^{\tau}+J_{t}+\operatorname{tr}\left[\tilde{\Lambda}(t)\left\{\tilde{U}^{\prime}(t) \tilde{U}(t)-I\right\}\right],
\end{aligned}
$$

where $\tilde{\Lambda}(t)$ is a symmetric matrix whose $(i, j)$-component is the Lagrange multiplier for that of the matrix constraints $\tilde{U}^{\prime}(t) \tilde{U}(t)=I$. Using (52), the differentiation of both sides of (63) with respect to $u_{i j}(t)$ is computed as

$$
\begin{aligned}
& \frac{\partial}{\partial u_{i j}(t)} L(\tilde{U}(t), \tilde{\Xi}(t), \tilde{\Lambda}(t)) \\
= & \frac{\partial}{\partial u_{i j}(t)}\left\{V_{t+1}^{\tau}+J_{t}\right\}+\frac{\partial}{\partial u_{i j}(t)} \operatorname{tr}\left[\tilde{\Lambda}(t)\left\{\tilde{U}^{\prime}(t) \tilde{U}(t)-I\right\}\right] \\
= & \operatorname{tr}\left[X(t) \frac{\partial Q_{t \mid t}}{\partial u_{i j}(t)}\right]+\operatorname{tr}\left[\tilde{\Lambda}(t)\left\{E_{j i} \tilde{U}(t)+\tilde{U}^{\prime}(t) E_{i j}\right\}\right] .
\end{aligned}
$$

Substituting (24) into the first term of the last expression in (64), we see

$$
\begin{aligned}
& \frac{\partial}{\partial u_{i j}(t)} L(\tilde{U}(t), \tilde{\Xi}(t), \tilde{\Lambda}(t)) \\
= & -\operatorname{tr}\left[X(t) Q_{t \mid t} N^{-1 / 2}\left\{E_{i j} \tilde{\Xi}(t) \tilde{U}^{\prime}(t)+\tilde{U}(t) \tilde{\Xi}(t) E_{j i}\right\} N^{-1 / 2} Q_{t \mid t}\right] \\
& +\operatorname{tr}\left[\tilde{\Lambda}(t)\left\{E_{j i} \tilde{U}(t)+\tilde{U}^{\prime}(t) E_{i j}\right\}\right] \\
= & -2 \operatorname{tr}\left[N^{-1 / 2} Q_{t \mid t} X(t) Q_{t \mid t} N^{-1 / 2} \tilde{U}(t) \tilde{\Xi}(t) E_{j i}\right] \\
& +2 \operatorname{tr}\left[\tilde{U}(t) \tilde{\Lambda}(t) E_{j i}\right] \\
= & -2\left[N^{-1 / 2} Q_{t \mid t} X(t) Q_{t \mid t} N^{-1 / 2} \tilde{U}(t) \tilde{\Xi}(t)\right]_{i j} \\
& +2[\tilde{U}(t) \tilde{\Lambda}(t)]_{i j}=0 .
\end{aligned}
$$

Namely, we have

$$
N^{-1 / 2} Q_{t \mid t} X(t) Q_{t \mid t} N^{-1 / 2} \tilde{U}(t) \tilde{\Xi}(t)=\tilde{U}(t) \tilde{\Lambda}(t) .
$$

Similarly, the derivative of (63) with respect to $\xi_{i}(t)$ is computed as

$$
\begin{aligned}
& \frac{\partial}{\partial \xi_{i}(t)} L(\tilde{U}(t), \tilde{\Xi}(t), \tilde{\Lambda}(t)) \\
= & \frac{\partial}{\partial \xi_{i}(t)}\left\{V_{t+1}^{\tau}+J_{t}\right\}+\frac{\partial}{\partial \xi_{i}(t)} \operatorname{tr}\left[\tilde{\Lambda}(t)\left\{\tilde{U}^{\prime}(t) \tilde{U}(t)-I\right\}\right] \\
= & \operatorname{tr}\left[X(t) \frac{\partial Q_{t \mid t}}{\partial \xi_{i}(t)}\right]+\frac{\partial}{\partial \xi_{i}(t)} \operatorname{tr}[\tilde{\Psi}(t) \tilde{\Xi}(t)] .
\end{aligned}
$$

Substituting (25) into the first term of the last expression in (67), we see

$$
\begin{aligned}
& \frac{\partial}{\partial \xi_{i}(t)} L(\tilde{U}(t), \tilde{\Xi}(t), \tilde{\Lambda}(t)) \\
& =\operatorname{tr}\left[X(t) \frac{\partial Q_{t \mid t}}{\partial \xi_{i}(t)}\right]+\frac{\partial}{\partial \xi_{i}(t)} \operatorname{tr}[\tilde{\Psi}(t) \tilde{\Xi}(t)] \\
& =-\operatorname{tr}\left[X(t) Q_{t \mid t} N^{-1 / 2} \tilde{U}(t) E_{i i} \tilde{U}^{\prime}(t) N^{-1 / 2} Q_{t \mid t}\right]+\psi_{i}(t) \\
& =-\operatorname{tr}\left[\tilde{U}^{\prime}(t) N^{-1 / 2} Q_{t \mid t} X(t) Q_{t \mid t} N^{-1 / 2} \tilde{U}(t) E_{i i}\right]+\psi_{i}(t) \\
& =-\left[\tilde{U}^{\prime}(t) N^{-1 / 2} Q_{t \mid t} X(t) Q_{t \mid t} N^{-1 / 2} \tilde{U}(t) E_{i i}\right]_{i i}+\psi_{i}(t) \\
& =0,
\end{aligned}
$$

which implies

$$
\tilde{U}^{\prime}(t) N^{-1 / 2}(t) Q_{t \mid t} X(t) Q_{t \mid t} N^{-1 / 2}(t) \tilde{U}(t)=\tilde{\Psi}(t) .
$$

Note that we have $\tilde{\Psi}(t) \tilde{\Xi}(t)=\tilde{U}(t) \tilde{\Lambda}(t)$ from (66) multiplied by $\tilde{U}^{\prime}(t)$ from left and (69). Hence, we have (16) from (66) since $\tilde{\Xi}(t)>0$. This completes the proof. 


\section{Numerical Algorithm}

In this section, we develop a method of computation of the solution $\left(Q_{t \mid t}, X(t), \tilde{U}(t), \tilde{\Xi}(t)\right), t=1,2, \cdots, \tau-1$ of the following set of equations for given the initial value $Q_{0 \mid 0}=Q^{0}$ and the terminal value $Q_{\tau \mid \tau}=Q\left(Q^{0}>Q\right)$.

$$
\begin{gathered}
N^{-1 / 2} Q_{t \mid t} X(t) Q_{t \mid t} N^{-1 / 2} \tilde{U}(t)=\tilde{U}(t) \tilde{\Psi}, \\
\left\{\begin{array}{c}
X(t)=F^{\prime}(t+1) X(t+1) F(t+1)+M, \\
X(\tau) \triangleq Q^{-1} N^{1 / 2} \tilde{U}(\tau) \tilde{\Psi} \tilde{U}^{\prime}(\tau) N^{1 / 2} Q^{-1}, \\
F(t) \triangleq Q_{t \mid t} Q_{t \mid t-1}^{-1} A,
\end{array}\right.
\end{gathered}
$$

and

$$
\left\{\begin{array}{l}
Q_{t \mid t-1}=A Q_{t-1 \mid t-1} A^{\prime}+G G^{\prime}, \\
Q_{t \mid t}{ }^{-1}=Q_{t \mid t-1}^{-1}+N^{-1 / 2} \tilde{U}(t) \tilde{\Xi}(t) \tilde{U}^{\prime}(t) N^{-1 / 2},
\end{array}\right.
$$

In what follows we always assume $\tilde{m}=m=n$ and $\tilde{\Psi} \neq I$. In this case, $\tilde{U}(t)$ is a square orthogonal matrix such that $\tilde{U}(t) \tilde{U}^{\prime}(t)=\tilde{U}^{\prime}(t) \tilde{U}(t)=I$. Hence, (16) implies

$$
X(t)=Q_{t \mid t}{ }^{-1} N^{1 / 2} \tilde{U}(t) \tilde{\Psi} \tilde{U}^{\prime}(t) N^{1 / 2} Q_{t \mid t}{ }^{-1},
$$

Multiplying $\tilde{\Psi}^{1 / 2} \tilde{U}^{\prime}(t) N^{1 / 2}$ from left and $N^{1 / 2} \tilde{U}(t) \tilde{\Psi}^{1 / 2}$ from right on the both sides of (71), we have

$$
\begin{aligned}
& \tilde{\Psi}^{1 / 2} \tilde{U}^{\prime}(t) N^{1 / 2} X(t) N^{1 / 2} \tilde{U}(t) \tilde{\Psi}^{1 / 2} \\
= & \left\{\tilde{\Psi}^{1 / 2} \tilde{U}^{\prime}(t) N^{1 / 2} Q_{t \mid t}{ }^{-1} N^{1 / 2} \tilde{U}(t) \tilde{\Psi}^{1 / 2}\right\}^{2},
\end{aligned}
$$

namely

$$
\begin{aligned}
& \tilde{\Psi}^{1 / 2} \tilde{U}^{\prime}(t) N^{1 / 2} Q_{t \mid t}^{-1} N^{1 / 2} \tilde{U}(t) \tilde{\Psi}^{1 / 2} \\
= & \left\{\tilde{\Psi}^{1 / 2} \tilde{U}^{\prime}(t) N^{1 / 2} X(t) N^{1 / 2} \tilde{U}(t) \tilde{\Psi}^{1 / 2}\right\}^{1 / 2} .
\end{aligned}
$$

Thus, we have shown

$$
\begin{aligned}
& Q_{t \mid t}{ }^{-1}=N^{-1 / 2} \tilde{U}(t) \tilde{\Psi}^{-1 / 2} \\
& \times\left\{\tilde{\Psi}^{1 / 2} \tilde{U}^{\prime}(t) N^{1 / 2} X(t) N^{1 / 2} \tilde{U}(t) \tilde{\Psi}^{1 / 2}\right\}^{1 / 2} \tilde{\Psi}^{-1 / 2} \tilde{U}^{\prime}(t) N^{-1 / 2},
\end{aligned}
$$

or, equivalently,

$$
\begin{aligned}
& Q_{t \mid t}=N^{1 / 2} \tilde{U}^{\prime}(t) \tilde{\Psi}^{1 / 2} \\
& \times\left\{\tilde{\Psi}^{1 / 2} \tilde{U}^{\prime}(t) N^{1 / 2} X(t) N^{1 / 2} \tilde{U}(t) \tilde{\Psi}^{1 / 2}\right\}^{-1 / 2} \tilde{\Psi}^{1 / 2} \tilde{U}(t) N^{1 / 2} .
\end{aligned}
$$

Let us consider a sequence of positive-definite symmetric matrices $\left\{Q_{|1|}, Q_{2 \mid 2}, \cdots, Q_{\tau-1 \mid \tau-1}\right\}$ for which we have $Q_{t \mid t}<Q_{t \mid t-1}$ and $Q_{\tau \mid \tau-1}>Q_{\tau \mid \tau}=Q$. Then, we can determine $\left\{Q_{2 \mid 1}, Q_{3 \mid 2}, \cdots, Q_{\tau \mid \tau-1}\right\}, \quad\{(\tilde{U}(t), \tilde{\Xi}(t)) ; t=1,2, \cdots$, $\tau-1, \tau\}$ and $\{X(1), X(2), \cdots, X(\tau-1), X(\tau)\}$ uniquely by $(70)_{1},(70)_{2}$ and (17), respectively. This means that the value of the right-hand side of (74) or (75) is uniquely determined as a function of $\left\{Q_{1 \mid 1}, Q_{2 \mid 2}, \cdots, Q_{\tau-1 \mid \tau-1}\right\}$, i.e.,

$$
Q_{t \mid t}=\Phi\left(t, Q_{|| 1}, Q_{2 \mid 2}, \cdots, Q_{\tau-1 \mid \tau-1}\right) .
$$

Setting $Q_{1}^{\tau-1} \triangleq\left[\begin{array}{llll}Q_{1 \mid 1} & Q_{2 \mid 2} & \cdots & Q_{\tau-1 \mid \tau-1}\end{array}\right]$ for simplicity, we can write

$$
\begin{aligned}
& Q_{1}^{\tau-1}=\left[\begin{array}{llll}
Q_{|| 1} & Q_{2 \mid 2} & \cdots & Q_{\tau-1 \mid \tau-1}
\end{array}\right] \\
& =\left[\Phi\left(1, Q_{1}^{\tau-1}\right) \Phi\left(2, Q_{1}^{\tau-1}\right) \cdots \Phi\left(\tau-1, Q_{1}^{\tau-1}\right)\right],
\end{aligned}
$$

Thus, the condition of optimality (16) requires $Q_{1}^{\tau-1}$ is a fixed-point with respect to the functions of $Q_{1}^{\tau-1}$ given by the right hand-side of (75). Hence, it is seen that we can apply the fixed-point iteration method

$$
Q_{1(i+1)}^{\tau-1}=\left[\Phi\left(1, Q_{1(i)}^{\tau-1}\right) \Phi\left(2, Q_{1(i)}^{\tau-1}\right) \cdots \Phi\left(\tau-1, Q_{1(i)}^{\tau-1}\right)\right],
$$

in order to get the solution.

\section{Numerical Examples}

In this section, we will show the results of numerical calculations for a simple 3-dimensional system and the two-step case: $\tau=2$.

Example $1 \quad(n=m=\tilde{m}=3)$. Let us consider a 3-dimensional system with

$$
\begin{gathered}
A=\left[\begin{array}{lll}
0.5 & 0.3 & 0.1 \\
0.2 & 0.4 & 0.2 \\
0.1 & 0.5 & 0.6
\end{array}\right], \quad G=\left[\begin{array}{ccc}
0.5 & 0 & 0 \\
0 & 0.5 & 0 \\
0 & 0 & 0.5
\end{array}\right], \\
M=150.0\left[\begin{array}{lll}
14.460 & 16.764 & 9.361 \\
16.764 & 33.371 & 25.688 \\
18.723 & 16.764 & 24.090
\end{array}\right] .
\end{gathered}
$$

$$
\begin{aligned}
R_{0} & =\left[\begin{array}{ccc}
-\frac{1}{8} & -\frac{3 \sqrt{3}}{8} & \frac{3}{4} \\
-\frac{3 \sqrt{3}}{8} & \frac{5}{8} & \frac{\sqrt{3}}{4} \\
-\frac{3}{4} & -\frac{\sqrt{3}}{4} & -\frac{1}{2}
\end{array}\right]\left[\begin{array}{ccc}
0.49 & 0 & 0 \\
0 & 1.0 & 0 \\
0 & 0 & 1.44
\end{array}\right]\left[\begin{array}{ccc}
-\frac{1}{8} & -\frac{3 \sqrt{3}}{8} & -\frac{3}{4} \\
-\frac{3 \sqrt{3}}{8} & \frac{5}{8} & -\frac{\sqrt{3}}{4} \\
\frac{3}{4} & \frac{\sqrt{3}}{4} & -\frac{1}{2}
\end{array}\right] \\
& =\left[\begin{array}{rrr}
1.2395 & 0.1015 & -0.2128 \\
0.1015 & 0.8673 & -0.3437 \\
-0.2128 & -0.3437 & 0.8231
\end{array}\right],
\end{aligned}
$$

i.e.,

$\tilde{\Psi}=\Psi=\operatorname{diag}(0.49,1.0,1.44), \quad \tilde{\Gamma}=\Gamma=\left[\begin{array}{ccc}-\frac{1}{8} & -\frac{3 \sqrt{3}}{8} & \frac{3}{4} \\ -\frac{3 \sqrt{3}}{8} & \frac{5}{8} & \frac{\sqrt{3}}{4} \\ -\frac{3}{4} & -\frac{\sqrt{3}}{4} & -\frac{1}{2}\end{array}\right]$.

For this case, the optimal observation gain matrix for the stationary Kalman filter is obtained as shown in Table 1 by applying the algorithm proposed by the author. Since $\tau=2, \tau-1=1$. Hence, we see from (77) that

$$
Q_{1}^{\tau-1}=Q_{\| \mid 1}=\Phi\left(1, Q_{\| 1}\right) .
$$

The fixed-point iteration is simply given by

$$
\begin{gathered}
Q_{2 \mid 1}^{(i)}=A Q_{|| 1}^{(i)} A^{\prime}+G G^{\prime}, \\
\tilde{U}_{i}(2) \tilde{\Xi}_{i}(2) \tilde{U}_{i}^{\prime}(2)=N^{1 / 2}\left(Q^{-1}-Q_{2 \mid 1}^{(i)-1}\right) N^{1 / 2}, \\
X_{i}(2)=Q^{-1} N^{1 / 2} \tilde{U}_{i}(2) \tilde{\Psi} \tilde{U}_{i}^{\prime}(2) N^{1 / 2} Q^{-1},
\end{gathered}
$$




$$
\begin{gathered}
X_{i}(1)=A^{\prime} Q_{2 \mid 1}^{(i)-1} Q X_{i}(2) Q Q_{2 \mid 1}^{(i)-1} A+M, \\
\tilde{U}_{i}(1) \tilde{\Xi}_{i}(1) \tilde{U}_{i}^{\prime}(1)=N^{1 / 2}\left(Q_{1 \mid 1}^{(i)-1}-Q_{1 \mid 0}^{-1}\right) N^{1 / 2}, \\
Q_{1 \mid 1}^{(i+1)}=N^{1 / 2} \tilde{U}_{i}^{\prime}(1) \tilde{\Psi}^{1 / 2} \\
\times\left\{\tilde{\Psi}^{1 / 2} \tilde{U}_{i}^{\prime}(1) N^{1 / 2} X_{i}(1) N^{1 / 2} \tilde{U}_{i}(1) \tilde{\Psi}^{1 / 2}\right\}^{-1 / 2} \tilde{\Psi}^{1 / 2} \tilde{U}_{i}(1) N^{1 / 2} .
\end{gathered}
$$

Table 1 . The result of the stationary problem.

\begin{tabular}{|c|c|}
\hline Variable & $n=m=\tilde{m}=3$ \\
\hline \multirow{4}{*}{$Q$} & {$\left[\begin{array}{rrr}0.044706 & -0.037105 & 0.021393 \\
-0.037105 & 0.063409 & -0.049670 \\
0.021393 & -0.049670 & 0.053837\end{array}\right]$} \\
\hline \multirow{U}{*}{} & {$\left[\begin{array}{rrr}0.36829 & 0.81593 & 0.44566 \\
0.72721 & 0.04584 & -0.68488 \\
0.57925 & -0.57633 & 0.57647\end{array}\right]$} \\
\hline$\tilde{\Xi}$ & $\operatorname{diag}\{193.4370,32.4391,3.7612\}$ \\
\hline \multirow{3}{*}{$X$} & {$\left[\begin{array}{rrr}2172.21 & 2516.12 & 1404.71 \\
2516.12 & 5008.24 & 3855.89 \\
1404.71 & 3855.89 & 3617.57\end{array}\right]$} \\
\hline \multirow{3}{*}{$H_{0}^{-1} H$} & {$\left[\begin{array}{rrr}48.5812 & 51.8729 & 26.9789 \\
51.8729 & 104.1273 & 79.1401 \\
26.9789 & 79.1401 & 76.9287\end{array}\right]$} \\
\hline \multirow{2}{*}{$H$} & {$\left[\begin{array}{rrr}-1.792323 & -0.480043 & 3.84318 \\
-5.682519 & 4.071498 & 2.19226 \\
0.158348 & 5.993808 & 4.98013\end{array}\right]$} \\
\hline$J$ & 232.2424463553761 \\
\hline
\end{tabular}

Table 2. The result of 2-step injection problem.

\begin{tabular}{|c|c|}
\hline Variable & $n=m=\tilde{m}=3$ \\
\hline$Q_{0}=Q_{0 \mid 0}$ & $0.5 \cdot I$ \\
\hline$\tilde{U}(1)$ & {$\left[\begin{array}{rrr}0.37097 & 0.81465 & 0.44577 \\
0.81465 & 0.04805 & -0.68935 \\
0.58300 & -0.57795 & 0.57103\end{array}\right]$} \\
\hline$\tilde{\Xi}(1)$ & $\operatorname{diag}\{136.5958,33.4742,5.6016\}$ \\
\hline \multirow{2}{*}{$Q_{|| 1}$} & {$\left[\begin{array}{rrr}0.040721 & -0.299750 & 0.016300 \\
-0.299750 & 0.054534 & -0.042525 \\
0.016300 & -0.042525 & 0.047350\end{array}\right]$} \\
\hline$\tilde{U}(2)$ & {$\left[\begin{array}{rrr}0.36831 & 0.81591 & 0.44569 \\
0.72717 & 0.04588 & -0.68492 \\
0.57928 & -0.57635 & 0.57641\end{array}\right]$} \\
\hline$\tilde{\Xi}(2)$ & $\operatorname{diag}\{193.4669,32.4389,3.7603\}$ \\
\hline & {$\left[\begin{array}{rrr}0.044706 & -0.037105 & 0.021393 \\
-0.037105 & 0.063409 & -0.049670 \\
0.021393 & -0.049670 & 0.053837\end{array}\right]$} \\
\hline
\end{tabular}

For the case of the initial error covariance $Q_{010}=Q^{0}=$ $0.5 I>Q$, we carried out the fixed point iteration given by (80)-(85). We got convergence only in 17 steps. The result is summarized in Table 2. By this example, one can see that the performance of the proposed algorithm of the fixed-point iteration is very good, and may be applicable for the case $\tau>2$.

\section{Concluding Remarks}

In this paper, we gave the numerical algorithm for the case that $\tilde{m}=m=n$. The numerical example is shown for $\tau=2$. The results for a more general case in which $\tilde{m}<n$ or $\tau>2$ will be reported in the near future.

\section{References}

[1] M. Athans, "On the determination of optimal costly measurement strategies for linear stochastic systems," Prep. 5th IFAC World Congress, Paris, 1/11, 1972.

[2] A. Logothetis, A. Isaksson and E. Skadas, "On sensor scheduling via information theoretic criteria," Prep. ACC, 1999.

[3] M. Aoki, and M. T. Li, "Optimal discrete time control system with cost for observation," IEEE Trans. Automatic Control, AC-14, 2, 165/175, 1969.

[4] A. Sano and M. Terao, "Measurement optimization in optimal process control," Automatica, 5, 705/714, 1970.

[5] S. Ihara, "Coding theory in white Gaussian channel with feedback," J. Multi-variate Analysis, 4, 74/87, 1974.

[6] Y. Takeuchi, "The optimal transmission of non-Gaussian signals through a noisy channel with feedback," IEEE Trans. Inform. Theory, IT-40, 5, 1624/1629, 1994.

[7] Y. Takeuchi and A. Ida, "Optimal Transmission of a set of Gaussian Signals through Discrete-Time Channels with Feedback," Proc. of 35th ISCIE SSS, Ube, Oct. 30-31, 2003, 118/123, 2004

[8] Y. Takeuchi, "An information theoretic approach to optimization of linear observations for the Kalman-Bucy filter," International Journal of Innovative Computing, Information \& Control, 1-3, 401/416, 2005.

[9] Y. Takeuchi, "Optimal transmission of a set of discrete-time Gaussian signals through channels with feedback," International Journal of Innovative Computing, Information \& Control, 2-5, 927/942, 2006.

[10] Y. Takeuchi, "Optimization of linear observations for the stationary Kalman filter based on a generalized Water Filling Theorem," International Journal of Innovative Computing, Information \& Control, 4-1, 211/230, 2008.

[11] Y. Takeuchi and A. Hirata, "A method of optimization of linear observations for the Kalman filter based on a generalized Water Filling Theorem," International Journal of Innovative Computing, Information \& Control, 5-1, 75/85, 2009.

[12]Y. Takeuchi and M. Inoue, "Optimization of observations for LQG control systems by an information theoretic approach," International Journal of Innovative Computing, 
Information \& Control, 6-1, 75/87, 2010.

[13]Y. Takeuchi, "Optimization of linear observations for the stationary Kalman filter under a quadratic performance criterion," International Journal of Innovative Computing, Information \& Control, 7-1, 85/99, 2011.

[14]Y. Takeuchi, "Optimization of the observations for stationary LQG stochastic control systems under a quadratic criterion," International Journal of Innovative Computing, Information \& Control, 8-3, 2313/2328, 2012.

[15]Y. Takeuchi and R. Matsumi, "Hybrid optimization of the observations for stationary LQG control systems," Trans. ISCIE, 25-12, 366/374, 2012.

[16] Y. Takeuchi and H. Nakai, "A New Method of Optimization of Linear Observations for the Stationary Kalman Filter," Proc. of 44th ISCIE SSS, Tokyo, Nov. 1-2, 2012, 21/28, 2013.
[17] Y. Takeuchi and H. Nakai, "An Improved Method of Optimization of Linear Observations for the Stationary Kalman Filter," Proc. of 45th ISCIE SSS, Okinawa, Nov. 1-2, 2013, 1/8, 2014.

[18] Y. Takeuchi, "Optimization of Linear Observations for the Stationary Kalman-Bucy Filter," Trans. ISCIE, 28-12, 467/475, 2015.

[19] Y. Takeuchi, "A New Algorithm of Optimization of Continuous-Time Linear Observations for the Stationary Kalman-Bucy Filter", Proc. of 47th ISCIE SSS, Honolulu, Dec. 5-8, 2015, 232/238, 2016.

[20] S. Kodama, and N. Suda, Matrix Theory for Systems Control (in Japanese), SICE, 1978.

[21] V. Kučera, "A contribution to matrix quadratic equations," IEEE Trans. Automatic Control, AC-17, 344/347, 1972. 